\title{
Cantilever beam electrostatic MEMS actuators beyond pull-in
}

\author{
Subrahmanyam Gorthi ${ }^{1}$, Atanu Mohanty ${ }^{1}$ and \\ Anindya Chatterjee ${ }^{2}$ \\ ${ }^{1}$ Supercomputer Education and Research Centre, Indian Institute of Science, \\ Bangalore 560012 , India \\ ${ }^{2}$ Mechanical Engineering, Indian Institute of Science, Bangalore 560 012, India \\ E-mail: subrahmanyam.gorthi@gmail.com, amohanty@serc.iisc.ernet.in and \\ anindya100@gmail.com
}

Received 25 May 2006, in final form 28 June 2006

Published 26 July 2006

Online at stacks.iop.org/JMM/16/1800

\begin{abstract}
The operational range of electrostatic MEMS parallel plate actuators can be extended beyond pull-in in the presence of an intermediate dielectric layer, which has a significant effect on the behavior of such actuators. Here, we study the behavior of cantilever beam electrostatic actuators beyond pull-in using a beam model along with a dielectric layer. The results from the simple beam model are validated with 3D simulations performed in CoventorWare $^{\mathrm{TM}}$. Three possible static configurations of the beam are identified over the operational voltage range. We call them floating, pinned and flat; the latter two are also called arc-type and S-type in the literature.

We compute the voltage ranges over which the three configurations can exist and the points where transitions occur between these configurations.

Voltage ranges are identified where bi-stable and tri-stable states exist. A classification of all possible transitions (pull-in and pull-out as well as transitions we term pull-down and pull-up) is presented based on the dielectric layer parameters. Dynamic stability analyses are presented for the floating and pinned configurations. For high dielectric layer thickness, discontinuous transitions between configurations disappear and the actuator has smooth predictable behavior, but at the expense of lower overall tunability.
\end{abstract}

(Some figures in this article are in colour only in the electronic version)

\section{Introduction}

This paper presents a study of cantilever beam electrostatic MEMS actuators beyond pull-in. The effects of an intermediate dielectric layer on possible configurations of the actuator and transitions between them are studied.

The behavior of electrostatic MEMS parallel plate actuators before pull-in is studied extensively in the literature [1-8]. These actuators can be meaningfully modeled beyond pull-in in the presence of an intermediate dielectric layer between the electrodes. Many MEMS devices operate beyond pull-in, e.g., capacitive switches $[9,10]$, zipper varactors $[11,12]$ and tunable CPW resonators [13].
Simple lumped element models of MEMS actuators with a single degree of freedom [14-17] result in easy calculations but fail to capture details of the behavior beyond pull-in. At the other end of modeling complexities, simulations of MEMS actuators beyond pull-in have been done using 3D models [18]. Similar approaches have been used in studying the hysteresis characteristics of electrostatic actuators [19]. 3D models $[2,18,19]$ lead to a detailed and accurate prediction, but simulations are expensive in time and computation, particularly for problems involving mechanical contacts. In this paper we employ a $1 \mathrm{D}$ analysis that, at an intermediate level of complexity, gives useful results with reasonable effort. The results with the $1 \mathrm{D}$ beam model are validated with $3 \mathrm{D}$ simulations in CoventorWare ${ }^{\mathrm{TM}}$. 


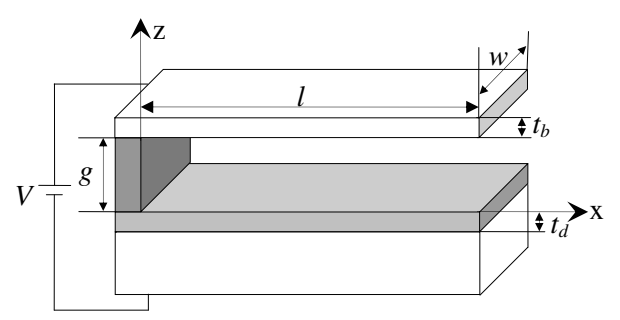

Figure 1. Schematic view of the cantilever beam electrostatic actuator.

This paper proceeds as follows. We discuss the possible equilibrium configurations of the beam actuator. Of these, two stable configurations exist beyond pull-in, denoted as pinned (arc-type) and flat (S-type) configurations [20, 21]. The beam with free end not touching the dielectric surface is called floating. The governing equations and the details of our finite-difference scheme are presented for the three configurations. Solutions show that multiple stable configurations can coexist. Transition points between configurations are computed. Dynamic stability analyses are conducted to further understand the nature of solutions and the behavior of the system. Finally, the results are validated with 3D simulations using CoventorWare ${ }^{\mathrm{TM}}$.

The main contribution of this paper is a classification of all possible transitions along with the associated dielectric layer thickness.

\section{Possible configurations}

The cantilever beam electrostatic actuator is illustrated in figure 1. It has three possible configurations in the entire operational range. These configurations differ under the boundary conditions at the free end of the cantilever beam and are as follows.

(i) Floating configuration. The cantilever beam has no contact with the dielectric layer and is illustrated in figure 2(a). The bending moment and shear force at the free end are zero.

(ii) Pinned configuration. The free end of the cantilever beam touches the dielectric layer but is free to pivot about the contact point and is illustrated in figure $2(b)$. The deflection (measured from the dielectric layer) and the bending moment are zero at the touching end.

(iii) Flat configuration. A non-zero length of the beam is in contact with the dielectric layer, as illustrated in figure 2(c). The contact length of the cantilever beam
Table 1. Boundary conditions at the free end of the cantilever beam for the three configurations.

\begin{tabular}{ll}
\hline Type of configuration & Boundary conditions \\
\hline Floating & $z^{\prime \prime}(l)=0 ; z^{\prime \prime \prime}(l)=0$ \\
Pinned & $z(l)=0 ; z^{\prime \prime}(l)=0$ \\
Flat & $z(a)=0 ; z^{\prime}(a)=0 ; z^{\prime \prime}(a)=0$ \\
\hline
\end{tabular}

varies with the applied voltage. Deflection measured from the dielectric layer, slope and moment are zero at the point separating the contact and the non-contact regions of the cantilever. The point is denoted by $x=a$ in figure 2(c). Note that, unlike the previous two configurations, the shear force is the only unknown boundary condition at this point. Here, the extent of the domain (the non-contacting region) is not known in advance; the previous two configurations represent boundary value problems while the flat configuration represents a free boundary problem. We will not concern ourselves unduly with this distinction, as will be seen below.

The boundary conditions discussed so far are summarized in table 1 . The parameter $l$ in the table denotes the length of the beam. At the clamped end of the cantilever beam, the boundary conditions are the same for all the configurations: the slope is zero and the deflection (measured from the dielectric layer) is equal to the zero bias height of the cantilever beam.

\section{Modeling and simulations}

The non-contact portion of the beam for all the three configurations is governed by the same equation.

\subsection{Governing equation}

The 1D equation governing the mechanical deformation of an Euler-Bernoulli beam is $[5,22]$

$$
E I \frac{\partial^{4} z}{\partial x^{4}}+\rho \frac{\partial^{2} z}{\partial t^{2}}=F_{e}
$$

where

$$
F_{e}=-\frac{\epsilon_{0} w V^{2}}{2\left(z+\frac{t_{d}}{\epsilon_{r}}\right)^{2}} .
$$

$F_{e}$ is the electrical force per unit length. The variables $x$ and $z$ in the above equations denote the position along the length and the lateral deflection of the beam, respectively, and $t$ is the time. Effects such as step-ups, stress-stiffening and softened

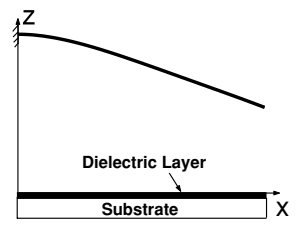

(a)

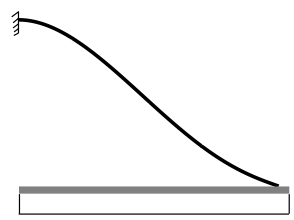

$(b)$

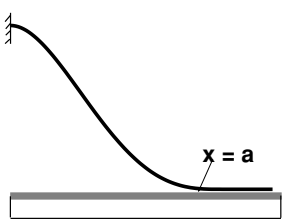

(c)

Figure 2. Possible configurations of the cantilever beam actuator. (The scale in the vertical direction is exaggerated.) (a) Floating configuration, $(b)$ pinned configuration and $(c)$ flat configuration. 
Table 2. Description of the parameters in the governing equation.

\begin{tabular}{ll}
\hline Parameter & Description \\
\hline$l$ & Beam length \\
$w$ & Beam width \\
$t_{b}$ & Beam thickness \\
$t_{d}$ & Dielectric layer thickness \\
$\rho$ & Mass per unit length of the beam \\
$g$ & Zero bias height of the cantilever beam \\
$I$ & Moment of inertia of the beam cross-section $\left(=\frac{w t_{b}^{3}}{12}\right)$ \\
$E$ & Young's modulus of the beam material \\
$\epsilon_{0}$ & Permittivity of free space \\
$\epsilon_{r}$ & Relative permittivity of the dielectric or \\
$V$ & dielectric constant \\
\hline
\end{tabular}

contact surfaces are not included in this model. The parameters in the governing equation are described in table 2.

The common practice [5] of using a fringing field correction such as $0.65 \frac{\left(z+t_{d} / \epsilon_{r}\right)}{w}$ is not adopted here. In the postpull-in regime, the cantilever beam has portions very close to the dielectric, where the fringing field is small. Further, neglecting the fringing field makes the analysis simpler and provides useful insights. Finally, calculations including the fringing field, though slightly complex, could if necessary be carried out using the approach adopted in this paper.

\subsection{Normalized equation}

The length quantities $x$ and $z$ (refer to figure 1) are normalized with respect to the length and zero bias height of the beam. The time $t$ is normalized with respect to a constant $T$, defined in such a way that the parameter $\rho$ in equation (1) becomes unity. The normalized quantities are as follows:

$$
\begin{gathered}
\hat{x}=\frac{x}{l}, \\
\hat{z}=\frac{z}{g}, \\
\hat{t}=\frac{t}{T}, \\
\text { where } \quad T=\sqrt{\frac{\rho l^{4}}{E I} .}
\end{gathered}
$$

Two other non-dimensional quantities are defined as follows:

$$
\begin{gathered}
\hat{V}=\sqrt{\frac{\epsilon_{0} w l^{4}}{2 E I g^{3}}} V, \\
h=\frac{t_{d}}{g \epsilon_{r}} .
\end{gathered}
$$

The governing equation becomes

$$
\frac{\partial^{4} \hat{z}}{\partial \hat{x}^{4}}+\frac{\partial^{2} \hat{z}}{\partial \hat{t}^{2}}=-\frac{\hat{V}^{2}}{(\hat{z}+h)^{2}} .
$$

For static analysis, there is no time dependence and the equation reduces to

$$
\frac{\mathrm{d}^{4} \hat{z}}{\mathrm{~d} \hat{x}^{4}}=-\frac{\hat{V}^{2}}{(\hat{z}+h)^{2}}
$$

The hats in the normalized equation are now dropped for convenience. Equation (10), with different right-end boundary conditions for each of the three configurations, is solved using finite differences. Some details follow.

\subsection{Finite difference approximation}

The derivatives are replaced by finite difference approximations, and the resulting system of algebraic equations is solved. Where boundary conditions involve derivatives, we introduce suitable fictitious points beyond the physical boundaries.

Specifically, a five-point central difference scheme is implemented because a fourth-order derivative is involved. Two fictitious points are introduced on each side of the noncontact length of the cantilever beam.

The non-contact length of the cantilever beam is divided into a uniform grid of $N$ points along the $x$-axis with a step size of $\Delta x$. These points are denoted by $x_{1}, x_{2}, \ldots, x_{N-1}, x_{N}$. Let $z_{j}$ denote the beam deflection at $x=x_{j}, j=1,2, \ldots, N$. Let the fictitious points be denoted by $z_{0}, z_{-1}$ on the left side and $z_{N+1}, z_{N+2}$ on the right side of the non-contact length with the same step size, $\Delta x$. Hence, totally $N+4$ points are considered along the $x$-axis. The finite difference approximations of derivatives of $z$ at $x=x_{j}$, derived from the five-point central difference scheme, are as follows:

$$
\begin{gathered}
z_{j}^{\prime}=\frac{z_{j-2}-8 z_{j-1}+8 z_{j+1}-z_{j+2}}{12(\Delta x)}, \\
z_{j}^{\prime \prime}=\frac{-z_{j-2}+16 z_{j-1}-30 z_{j}+16 z_{j+1}-z_{j+2}}{12(\Delta x)^{2}} \\
z_{j}^{\prime \prime \prime}=\frac{-z_{j-2}+2 z_{j-1}-2 z_{j+1}+z_{j+2}}{2(\Delta x)^{3}}, \\
z_{j}^{\prime \prime \prime \prime}=\frac{z_{j-2}-4 z_{j-1}+6 z_{j}-4 z_{j+1}+z_{j+2}}{(\Delta x)^{4}}
\end{gathered}
$$

for $j=1,2, \ldots, N$.

The governing equation (10) in terms of finite differences is

$$
z_{j-2}-4 z_{j-1}+6 z_{j}-4 z_{j+1}+z_{j+2}+\frac{(\Delta x)^{4} V^{2}}{\left(z_{j}+h\right)^{2}}=0 .
$$

The derivatives under the boundary conditions are also replaced by the finite difference approximations. The boundary conditions at the clamped end of the three configurations, written in terms of finite differences, are

$$
\begin{gathered}
z_{1}=1 \\
z_{-1}-8 z_{0}+8 z_{2}-z_{3}=0 .
\end{gathered}
$$

At the other end of the beam, the boundary conditions in terms of finite differences for the three configurations are as follows.

(i) Floating configuration.

$$
\begin{gathered}
-z_{N-2}+16 z_{N-1}-30 z_{N}+16 z_{N+1}-z_{N+2}=0 \\
-z_{N-2}+2 z_{N-1}-2 z_{N+1}+z_{N+2}=0 .
\end{gathered}
$$

(ii) Pinned configuration.

$$
\begin{gathered}
z_{N}=0 \\
-z_{N-2}+16 z_{N-1}-30 z_{N}+16 z_{N+1}-z_{N+2}=0 .
\end{gathered}
$$

(iii) Flat configuration.

$$
\begin{gathered}
z_{N}=0 \\
z_{N-2}-8 z_{N-1}+8 z_{N+1}-z_{N+2}=0 \\
-z_{N-2}+16 z_{N-1}-30 z_{N}+16 z_{N+1}-z_{N+2}=0
\end{gathered}
$$


The solution procedures adopted for the floating and pinned configurations are identical; the flat configuration has a minor addition.

In the floating and pinned configurations, the non-contact length is known and is equal to 1 for the normalized case. Hence, $z$ values at $N+4$ points are the only unknowns. Four boundary conditions are known in each of these two configurations. Equation (15) computed for $j=1,2, \ldots, N$ together with the four boundary conditions of the corresponding configuration form a system of $N+4$ nonlinear algebraic equations. The system of nonlinear equations is solved through the Newton-Raphson iterative technique [23], and $z$ values at $N+4$ points are computed.

For the flat configuration, any one solution determines all other solutions by a scaling law discussed in the following subsection. The procedure to find one solution, for which we assume that the non-contacting length is unity, is as follows. $V$ is initialized with an arbitrary value. The corresponding value of $z$ is computed using the three boundary conditions at the flat end (at $z_{N}$ ) and the zero-slope condition at the fixed end (at $z_{1}$ ). In other words, equation (16) is initially ignored. With $V$ given, this is enough to determine the solution. The computed value of $z_{1}$, at the fixed end, should be unity. The chosen $V$ is iteratively modified to match equation (16). Once this $V$ is found, all other flat solutions for the same $h$ can be found by scaling as discussed in the following subsection. All the numerical computations in this paper are done with $N=1001$ (1000 intervals).

This concludes our description of the finite-difference scheme.

The governing equation (10) includes a nonlinear term. The system can have multiple solutions, and the solution obtained depends on the initial guess made in the iterative procedure. Physically feasible solutions avoid interpenetration between the beam and the dielectric layer. Such physically feasible equilibrium solutions may or may not be dynamically stable. For the present, we concentrate on obtaining the equilibrium solutions (for stability analysis, see section 5).

\subsection{Scaling law in the flat configuration}

In the flat regime, a scaling law is found. Due to this scaling, if the solution is computed at one voltage, solutions at other voltages can be found by scaling without solving the governing equation again. Let

$$
\xi=x \sqrt{V}
$$

Equation (10) becomes

$$
\frac{\mathrm{d}^{4} z}{\mathrm{~d} \xi^{4}}=\frac{-1}{(z+h)^{2}} .
$$

Let the $\xi$ value at $x=a$ (see figure 2(c)) be denoted by $\xi_{0}$. The boundary conditions expressed in terms of $\xi$ are

$$
\begin{aligned}
& z(\xi=0)=1 \quad \text { and } \quad z^{\prime}(\xi=0)=0 \\
& z\left(\xi=\xi_{0}\right)=z^{\prime}\left(\xi=\xi_{0}\right)=z^{\prime \prime}\left(\xi=\xi_{0}\right)=0 .
\end{aligned}
$$

It is now evident from equations (26) and (27) that neither the governing equation nor the boundary conditions depend on the applied voltage. The value of $\xi_{0}$ and the function $z(\xi)$ for a given voltage can be computed from the numerical procedure described previously; they are fixed for a given $h$. Let $\alpha$ be the physical $(x)$ non-contact length of the beam for any $V$. Then,

$$
\alpha \sqrt{V}=\xi_{0}=\text { constant. }
$$

Similarly, $z(\xi)$ computed at one voltage can be used to compute $z(x)$ at all voltages. We note that this scaling approach is applicable even to models $[5,24]$ that account for the fringing field.

\section{Effects of the dielectric layer}

The governing equation (10) has only two parameters $V$ and $h$. $h$ is proportional to the dielectric thickness for a given zero bias height and dielectric constant. Similarly, $V$ is proportional to the applied voltage. We study solutions for fixed $h$ and varying $V$, for a range of values of $h$.

The following subsection gives details regarding voltage limits of the three possible configurations of the beam.

\subsection{Normalized voltage (V) limits of configurations}

(i) Floating configuration. The lower limit $\left(V_{\text {float }}^{\min }\right)$ is, trivially, zero. The upper limit $\left(V_{\text {float }}^{\text {max }}\right)$ is decided either by disappearance of solutions via a so-called turning point (for small $h$, as discussed later) or by (for large $h$ ) contact with the dielectric layer. Beyond this upper limit, the actuator switches to the pinned or flat configuration.

(ii) Pinned configuration. The end of the beam can, in principle, always be held pinned (say, by an external agent) against the dielectric layer by a suitable additional vertical force at the end point. If that force needs to act downward, then such a pinned solution is physically unfeasible because when we remove that force, the end point moves up. Upward acting forces are feasible because when we remove that force, the end point tends to move down and presses against the dielectric layer which, through mechanical contact, can apply an upward force. The lower limit $\left(V_{\text {pin }}^{\min }\right)$ is therefore the voltage at which the shear force at the contacting end of the cantilever beam becomes zero (and the boundary conditions of the pinned configuration are also satisfied). Below $V_{\text {pin }}^{\text {min }}$, the dielectric layer would have to mechanically pull down on the free end; since it cannot do so, the actuator is in the floating configuration. The upper limit $\left(V_{\text {pin }}^{\max }\right)$ is decided either by disappearance of solutions via a so-called turning point (for small $h$, as discussed later) or by (for large $h$ ) extended contact with the dielectric layer (zero slope at the end). Beyond this upper limit, the actuator switches to the flat configuration. The transition at $\left(V_{\mathrm{pin}}^{\max }\right)$ has not been elucidated in the literature and is one of post-pull-in insights offered in this paper.

(iii) Flat configuration. The lower limit $\left(V_{\text {flat }}^{\min }\right)$ is that at which the non-contacting length of the beam equals the total length of the beam. There is no upper $V$ limit for the flat configuration. The non-contacting length approaches zero as $V \rightarrow \infty$.

The $V$ limits for the three configurations, computed for varying $h$ values, are shown in figure 3 . Possible transitions between configurations are shown in figure 4 , based on figure 3 . 


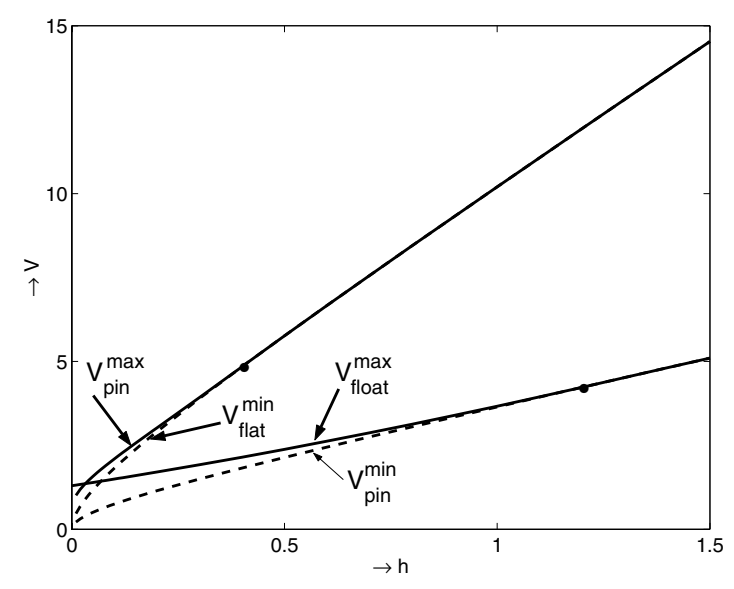

Figure 3. $V$ limits of the three configurations.

\subsection{Transitions}

Four transitions are identified, as suggested by figure 4 . The following insights into transitions from one configuration to another would be impossible with lumped parameter models and somewhat obscured with 3D models. These simple insights form one of the main benefits of the beam model for the actuator.

4.2.1. Pull-in. Pull-in occurs when the floating configuration solution disappears, as discussed earlier. Figure 5 shows the magnitude of the pull-in discontinuity for varying $h$ values. The jump in the height at the free end of the beam at the point of transition results from a turning-point bifurcation, as discussed later. Here, we note (figure 5) that the magnitude decreases essentially linearly with increasing $h$. It is interesting to note that for $h<0.03$ (figure $4(a)$ ), the transition from floating has to be to the flat configuration. For $0.03<h<0.07$ (figure $4(b)$ ), the transition could be to either the pinned or the flat configuration, and only a full nonlinear dynamic analysis (not attempted here) can resolve which configuration is reached immediately after pull-in. For $h>0.07$, the transition has to be to the pinned configuration. Upon increasing the voltage, regardless of $h$, any pinned configuration will transition to a flat configuration.

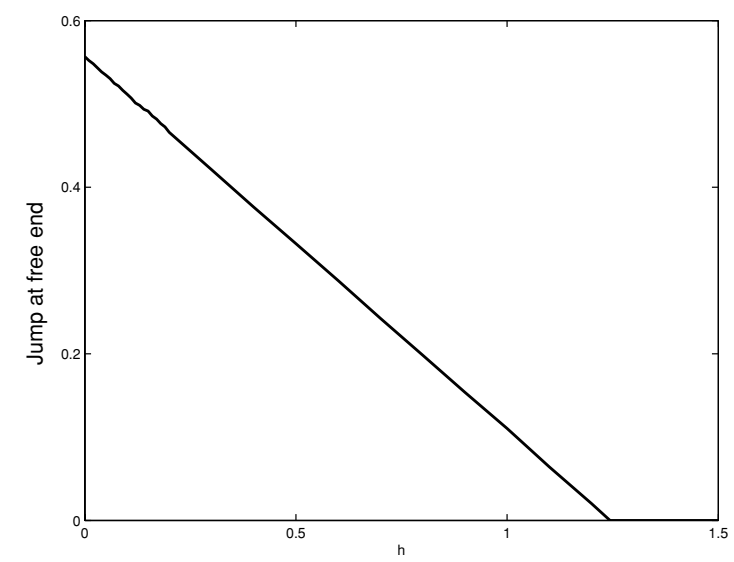

Figure 5. Variation of the magnitude of the pull-in discontinuity with $h$.

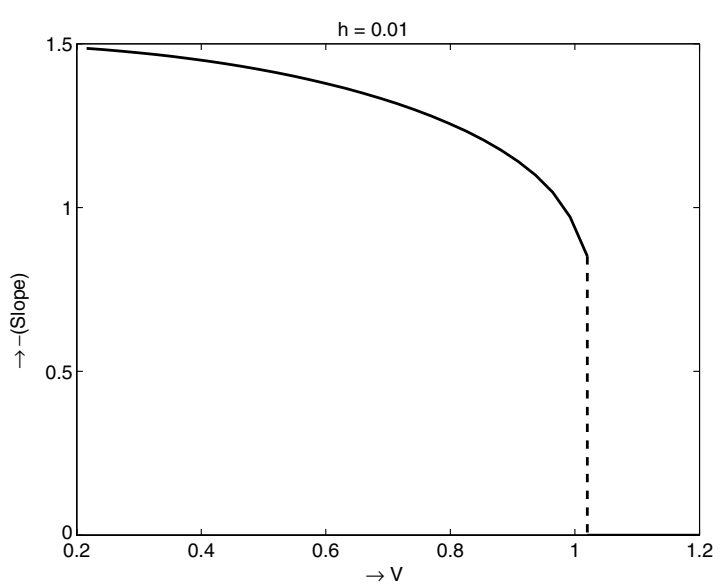

Figure 6. Pull-down: jump-in slope at the touching end of the beam.

4.2.2. Pull-down. The transition from the pinned to the flat configuration is referred to here as pull-down. The pinned configuration has a non-zero slope at the beam's end point while the flat configuration has a zero slope. As is the case for pull-in, a discontinuous transition from pinned to flat occurs due to a turning-point bifurcation, as discussed later. Figure 6 shows the pull-down discontinuity for $h=0.01$. As $V$ is

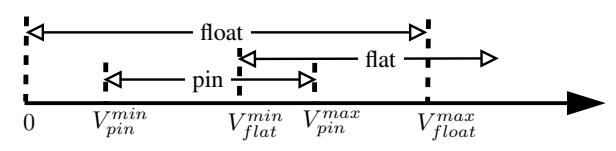

(a) $0<\mathrm{h}<0.03$

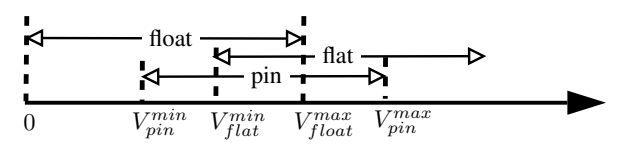

(b) $0.03<\mathrm{h}<0.07$

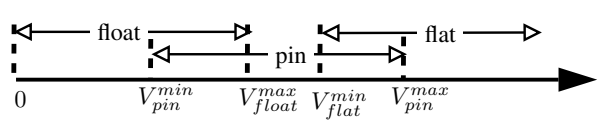

(c) $0.07<\mathrm{h}<0.40$

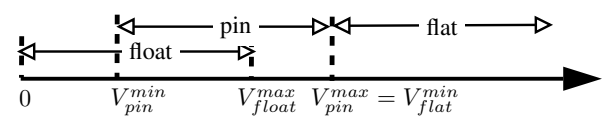

(d) $0.40<\mathrm{h}<1.24$

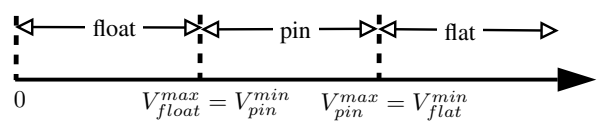

(e) $\mathrm{h}>1.24$

Figure 4. Classification of possible transitions based on the numerical results of figure 3 . 




Figure 7. Variation of the magnitude of the pull-down discontinuity with $h$.

increased from $V_{\text {pin }}^{\min }$, the magnitude of the slope at the touching end point decreases faster and faster until the curve turns around (not shown here; see the discussion later) and the pinned solution disappears. Figure 7 shows the magnitude of the pull-down slope discontinuity for different $h$. For $h>0.4$, the discontinuity in the transition disappears.

4.2.3. Pull-up. Starting in the flat configuration, a transition is possible to a pinned configuration. Here, we refer to such a transition as pull-up. Again, for $h>0.4$, pull-up is continuous (figure 4). In addition, for $h<0.07$, it is not clear without nonlinear dynamics analysis (not conducted here) whether the transition at $V_{\text {flat }}^{\min }$ occurs to the pinned or the floating configuration. Note that any pinned configuration must eventually transition to the floating configuration as $V$ is decreased.

4.2.4. Pull-out. Finally, the transition from either pinned or flat to floating is called pull-out. Note that, as is widely observed in experiments, pull-out does not in general occur at the same voltage as pull-in; however, for large enough $h$, it does. This consistency in physical behavior may be useful in applications.

The width of bi-stability regions between the floatingpinned and the pinned-flat configurations, as a function of $h$, is shown in figure 8 . There may be two or even three stable configurations at a given $V$. As $V$ is changed back and forth so that transitions occur between states, therefore, there can be hysteresis in the actuator's behavior. Note that there is no such bi-stability for $h>1.24$ and that there is tri-stability for $h<0.07$.

The existence of tri-stable states has not previously been noted for such actuators in the literature.

\section{Dynamic stability of equilibrium solutions}

\subsection{Method}

The dynamic stability of an equilibrium solution can be determined by considering small variations of that solution and is governed by an eigenvalue problem.

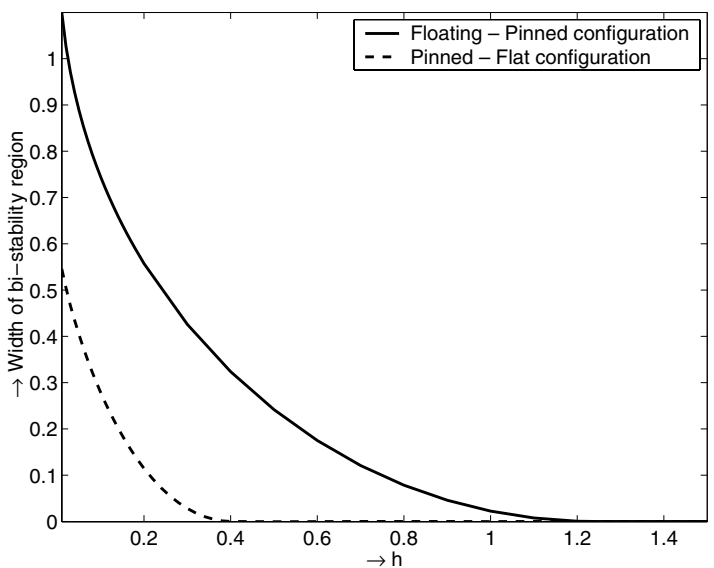

Figure 8. Variation of the width of the bi-stability region with $h$.

Let $z_{\text {eq }}$ be an equilibrium solution. Then,

$$
\frac{\partial^{4} z_{\mathrm{eq}}}{\partial x^{4}}=-\frac{V^{2}}{\left(z_{\mathrm{eq}}+h\right)^{2}} .
$$

Let $\zeta$ be a small perturbation to $z_{\text {eq }}$. Putting

$$
z=z_{\text {eq }}+\zeta
$$

in equation (9),

$$
\frac{\partial^{4}\left(z_{\mathrm{eq}}+\zeta\right)}{\partial x^{4}}+\frac{\partial^{2}\left(z_{\mathrm{eq}}+\zeta\right)}{\partial t^{2}}=-\frac{V^{2}}{\left(z_{\mathrm{eq}}+\zeta+h\right)^{2}} .
$$

Linearizing for small $\zeta$,

$$
\frac{\partial^{4}\left(z_{\mathrm{eq}}+\zeta\right)}{\partial x^{4}}+\frac{\partial^{2}\left(z_{\mathrm{eq}}+\zeta\right)}{\partial t^{2}}=-\frac{V^{2}}{\left(z_{\mathrm{eq}}+h\right)^{2}}+\frac{2 V^{2}}{\left(z_{\mathrm{eq}}+h\right)^{3}} \zeta
$$

By equation (29),

$$
\frac{\partial^{4} \zeta}{\partial x^{4}}+\frac{\partial^{2} \zeta}{\partial t^{2}}=\frac{2 V^{2}}{\left(z_{\text {eq }}+h\right)^{3}} \zeta
$$

The above system is discretized using a modal expansion along the lines of [25] by letting

$$
\zeta(x, t)=\sum_{i=1}^{n} a_{i}(t) \psi_{i}(x),
$$

where $\psi_{i}(x)$ is the $i$ th normal (or natural) mode of the beam in the absence of electrostatic forces and $a_{i}(t)$ is the associated modal coordinate or participation factor. Large $n$ gives high accuracy. The normal modes used are

$$
\psi_{i}(x)=\cosh \left(\lambda_{i} x\right)-\cos \left(\lambda_{i} x\right)-b_{i}\left(\sinh \left(\lambda_{i} x\right)-\sin \left(\lambda_{i} x\right)\right),
$$

where $\lambda_{i}$ and $b_{i}$ are as follows for the floating and pinned configurations.

(i) Floating. $\lambda_{i}$ is the $i$ th root of

$$
1+\cosh \left(\lambda_{i}\right) \cos \left(\lambda_{i}\right)=0
$$

and

$$
b_{i}=\frac{\sinh \left(\lambda_{i}\right)-\sin \left(\lambda_{i}\right)}{\cosh \left(\lambda_{i}\right)+\cos \left(\lambda_{i}\right)} .
$$


(ii) Pinned. $\lambda_{i}$ is the $i$ th root of

$$
\operatorname{coth}\left(\lambda_{i}\right)-\cot \left(\lambda_{i}\right)=0
$$

and

$$
b_{i}=\operatorname{coth}\left(\lambda_{i}\right)
$$

Stability analysis of the flat configuration is more difficult because the contacting length of the beam changes during motion. Such an analysis is not attempted here. Note, however, that an extended contact region suggests, intuitively, that the flat configuration is stable.

The normal modes are, as the name suggests, orthonormal:

$$
\int_{0}^{1} \psi_{i}(x) \psi_{j}(x) \mathrm{d} x= \begin{cases}1 & \text { if } i=j \\ 0 & \text { otherwise }\end{cases}
$$

From equation (34),

$$
\frac{\partial^{4} \psi_{i}(x)}{\partial x^{4}}=\lambda_{i}^{4} \psi_{i}(x)
$$

Equation (32) becomes

$$
\begin{aligned}
& \sum_{i=1}^{n}\left[\frac{\partial^{2} a_{i}(t)}{\partial t^{2}} \psi_{i}(x)+\lambda_{i}^{4} a_{i}(t) \psi_{i}(x)\right] \\
& \approx \frac{2 V^{2}}{\left(z_{\mathrm{eq}}+h\right)^{3}} \sum_{i=1}^{n} a_{i}(t) \psi_{i}(x)
\end{aligned}
$$

where we write ' $\approx$ ' instead of ' $=$ ' to emphasize that a finite- $n$ approximation is being made.

Multiplying the above with $\psi_{j}(x), j=1,2, \ldots, n$ and integrating over the length, we obtain

$$
\begin{aligned}
& \frac{\partial^{2} a_{j}(t)}{\partial t^{2}}+\lambda_{j}^{4} a_{j}(t) \\
& =\sum_{i=1}^{n} a_{i}(t) \int_{0}^{1} \frac{2 V^{2}}{\left(z_{\mathrm{eq}}+h\right)^{3}} \psi_{i}(x) \psi_{j}(x) \mathrm{d} x,
\end{aligned}
$$

where we have reintroduced ' $=$ ' instead of ' $\approx$ ' for convenience although the finite- $n$ approximation remains. Note, also, that the integral on the right-hand side requires knowledge of $z_{\mathrm{eq}}(x)$ from a separate calculation.

In a matrix form,

$$
\frac{\partial^{2} \vec{a}}{\partial t^{2}}+\Lambda^{4} \vec{a}-B \vec{a}=0
$$

where

$$
\vec{a}=\left[a_{1}(t), a_{2}(t), a_{3}(t), \ldots, a_{n}(t)\right]^{T},
$$

and $\Lambda$ is a diagonal matrix with $\Lambda_{j j}=\lambda_{j}$. Also, $B$ is a square matrix with

$$
B_{i j}=\int_{0}^{1} \frac{2 V^{2}}{\left(z_{\mathrm{eq}}+h\right)^{3}} \psi_{i}(x) \psi_{j}(x) \mathrm{d} x .
$$

Let $\vec{a}=\vec{u} \mathrm{e}^{\sigma t}$. Substituting in equation (43),

$$
\left(-\Lambda^{4}+B\right) \vec{u}=\sigma^{2} \vec{u} .
$$

The eigenvalues of the above system determine stability. By the symmetry of $\Lambda$ and $B$, all $\sigma^{2}$ are real. If all $\sigma^{2}$ are negative, then all solutions are linear combinations of sinusoidal oscillations, and the original equilibrium solution is dynamically stable. A positive $\sigma^{2}$ implies instability.
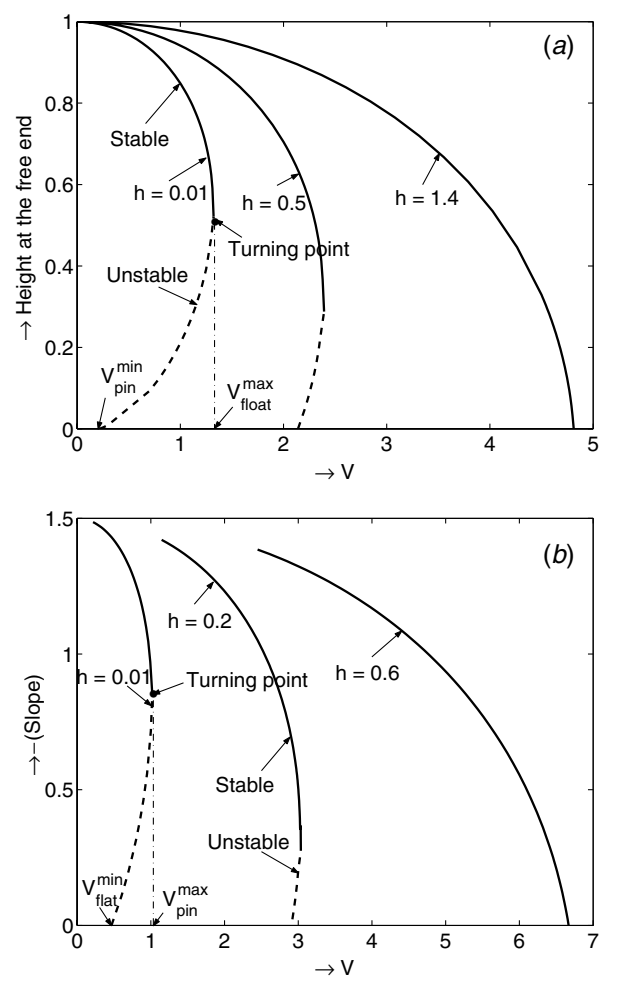

Figure 9. Stability analysis of equilibrium solutions of the normalized governing equation. (a) Floating configuration: variation of height at the free end of the beam with the applied voltage and $(b)$ pinned configuration: variation of slope at the pinned end with the applied voltage.

At a stability boundary, one eigenvalue will be $\sigma^{2}=0$ (a degenerate case).

Note that the numerical integration to be performed in equation (45) requires $z_{\mathrm{eq}}$ and $\psi$ values at the mesh points. Of these, $z_{\text {eq }}$ was computed above at a number of mesh points and $\psi$ was given above. All integrations were performed using Simpson's rule. Five normal modes were used in the stability results presented below. The stability boundary points obtained did not differ from those obtained with three and with seven modes (those calculations are not presented here).

\subsection{Stability results}

Figures $9(a)$ and $(b)$ show the results of stability analysis for the floating and pinned configurations, respectively. Each point on each curve represents an equilibrium solution (floating or pinned). Solid lines indicate stable and dashed lines indicate unstable solutions. Where the two branches coalesce at a turning point, the solution is borderline unstable by linear analysis. The value of $V$ at the turning point represents pullin and pull-down in the floating and pinned configurations, respectively.

\subsection{Connections with section 4}

The stability results plotted above also include families of equilibrium solutions plotted therein. It is useful to discuss these plots in light of section 4. 
(i) In figure $9(a)$, the turning points indicate $V_{\text {float }}^{\max }$. If there are no turning points (large $h$ ), intersection with the horizontal axis indicates $V_{\text {float }}^{\max }$. Whether a turning point exists or not, intersection with the horizontal axis always indicates a floating solution where the beam tip just contacts the dielectric surface; the same solution happens to be a pinned solution as well, with the contact force equal to zero. The corresponding $V$ is therefore $V_{\text {pin }}^{\min }$ (recall the discussion of section 4).

The above result, though probably not new, is apparently not well known in the MEMS community. We discuss it further, briefly, from a mechanical point of view.

If there is no turning point in the curve, then the slope of the curve is negative; this means that decreasing $V$ tends to raise the beam tip off the dielectric surface, following a stable solution curve. Thus, reducing $V$ causes disappearance of the pinned solution. Vice versa, increasing $V$ would tend to lower the beam tip into the dielectric surface, bringing a vertical contact force into play and producing a pinned solution. Hence, the intersection marks $V_{\text {pin }}^{\min }$.

On the other hand, if there is a turning point, then slightly raising $V$ produces a floating solution where the beam tip is slightly above the dielectric surface. However, that solution is unstable, and so a slight downward perturbation to it will cause the beam tip to come down further until it touches the dielectric surface, producing a pinned solution. Vice versa, lowering $V$ slightly would make the beam tip tend to dip below the dielectric surface; this would bring into play an upward acting contact force, disturbing the solution, and, the solution being unstable, would make the beam move further up, away from the pinned state.

In this way, simultaneous consideration of equilibrium and stability helps us understand why the intersection point of the floating solution curve with the horizontal axis indicates $V_{\text {pin }}^{\min }$.

(ii) Similarly, considering the pinned configuration illustrated in figure $9(b)$ (the vertical axis shows the slope at the end of the beam), we observe that $V_{\text {pin }}^{\max }$ is the maximum $V$ at which a stable solution exists (whether there is a turning point or not), and the solution curve intersects the horizontal axis at $V_{\text {flat }}^{\min }$.

(iii) For $h>0.4$, all pinned solutions are stable. In other words, $V_{\text {pin }}^{\max }=V_{\text {flat }}^{\min }$. Simultaneously, stable pinned and flat solutions no longer coexist (this mode of bi-stability disappears).

(iv) For $h>1.24$, all floating solutions are stable, and $V_{\text {float }}^{\max }=V_{\text {pin }}^{\min }$. Simultaneous multiple stable solutions of any kind no longer exist.

\section{Validation of results with the 3D FEM-BEM model in CoventorWare ${ }^{\mathrm{TM}}$}

3D simulations are performed in CoventorWare ${ }^{\mathrm{TM}}-2004$ and the results are compared to those of the 1D beam model. CoventorWare $^{\mathrm{TM}}$ uses finite element method (FEM) for solving the mechanical domain and boundary element method (BEM) for the electrical domain. Nonlinear effects are also

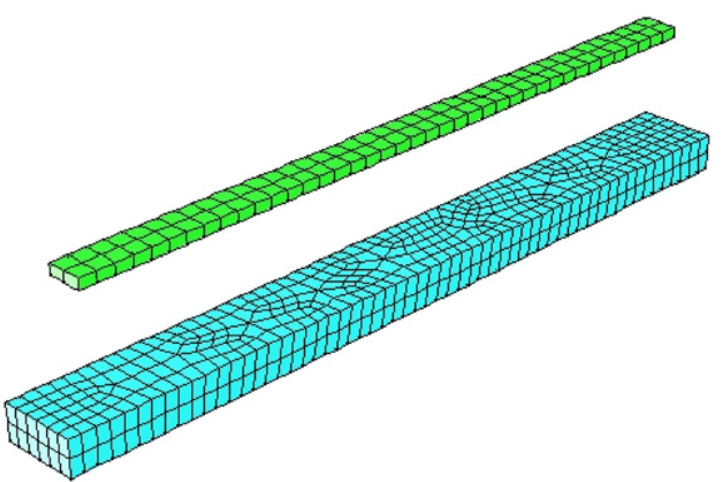

Figure 10. 3D model of the cantilever beam electrostatic actuator. (The $z$-scale is exaggerated by a factor of 10.)

Table 3. $h$ values that are considered to validate the classification.

\begin{tabular}{llllll}
\hline & Case 1 & Case 2 & Case 3 & Case 4 & Case 5 \\
\hline$h$ & 0.005 & 0.05 & 0.2 & 0.8 & 1.5 \\
\hline
\end{tabular}

Table 4. A comparison of the cases.

\begin{tabular}{|c|c|c|c|c|c|}
\hline & Case 1 & Case 2 & Case 3 & Case 4 & Case 5 \\
\hline Tri-stable states & $\sqrt{ }$ & $\sqrt{ }$ & $\times$ & $x$ & $\times$ \\
\hline $\begin{array}{l}\text { Bi-stable state: } \\
\text { floating-pinned }\end{array}$ & $\sqrt{ }$ & $\sqrt{ }$ & $\sqrt{ }$ & $\sqrt{ }$ & $\times$ \\
\hline $\begin{array}{l}\text { Bi-stable state: } \\
\text { pinned-flat }\end{array}$ & $\sqrt{ }$ & $\sqrt{ }$ & $\sqrt{ }$ & $x$ & $\times$ \\
\hline $\begin{array}{l}\text { Transition from } \\
\text { floating configuration } \\
\text { is to: }\end{array}$ & Flat & $\begin{array}{l}\text { Pinned/ } \\
\text { flat }\end{array}$ & Pinned & Pinned & Pinned \\
\hline
\end{tabular}

taken into account and the 3D model is shown in figure 10 . The qualitative classification is found to be applicable even for the 3D model. Further, the displacements, transition points between the configurations and contact lengths are in good agreement with the 1D model.

The dimensions of the moving electrode (upper electrode) are $400 \times 10 \mu \mathrm{m}^{2}$, with a thickness of $0.3 \mu \mathrm{m}$ and the material is polysilicon. The left side surface of this electrode is fixed. A zero bias gap ( $g$ ) of $3 \mu \mathrm{m}$ is taken for all the cases by defining a contact plane in between the moving and fixed electrodes, at a distance of $3 \mu \mathrm{m}$ from the moving electrode. The gap between the contact plane and the fixed electrode $\left(t_{d}\right)$ is varied to get different values of $h$. Table 3 shows the values of $h$ that are chosen to validate the classification. Note that the corresponding $t_{d}$ values are obtained by just multiplying $h$ with $g$ since $\epsilon_{r}=1$, as air is the dielectric medium here.

The moving electrode is meshed using the mesh type of Manhattan bricks and parabolic element order. Element sizes of 4,5 and $0.3 \mu \mathrm{m}$ are taken along the length, width and thickness respectively. The fixed electrode is surface meshed with an element size of 4 , with a quadrilateral type of elements and by using the Paving algorithm.

The key differences among these cases are highlighted in table 4 . The existence of tri-stable states at a given voltage 


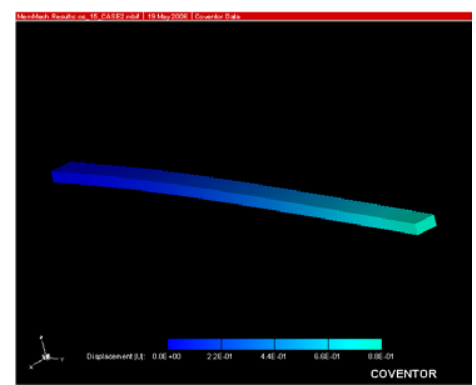

(a)

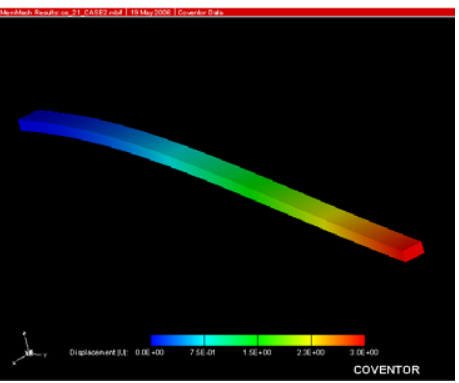

(b)

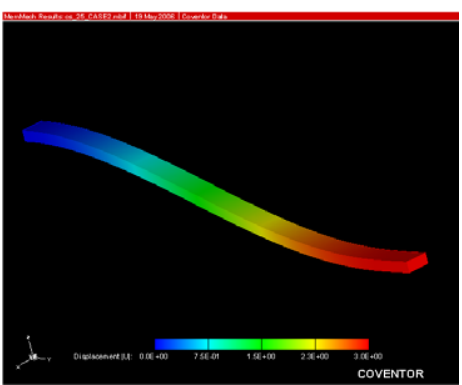

(c)

Figure 11. Tri-stable states for the Case 2 example at 1.5 V. (The $z$-scale is exaggerated by a factor of 15.) (a) Floating configuration, (b) pinned configuration and (c) flat configuration.

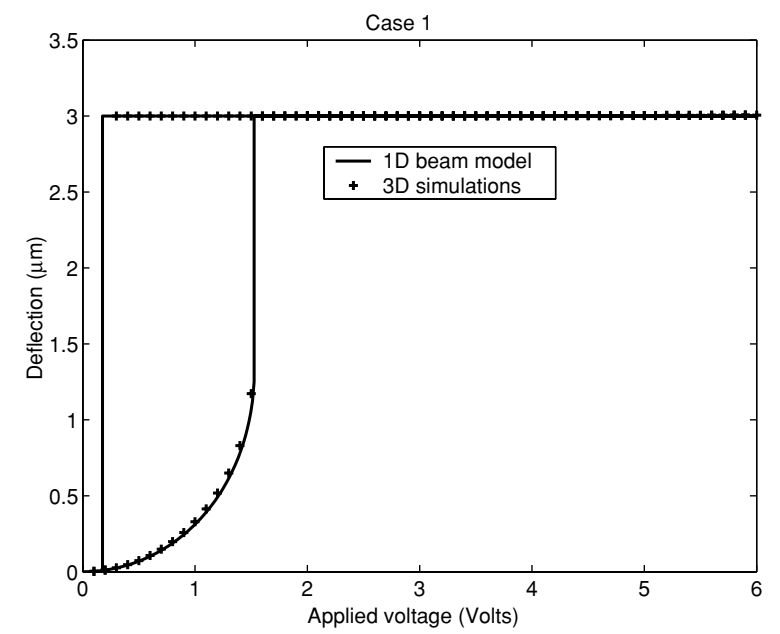

Figure 12. Variation of the moving electrode tip deflection with the applied voltage for Case 1 example.

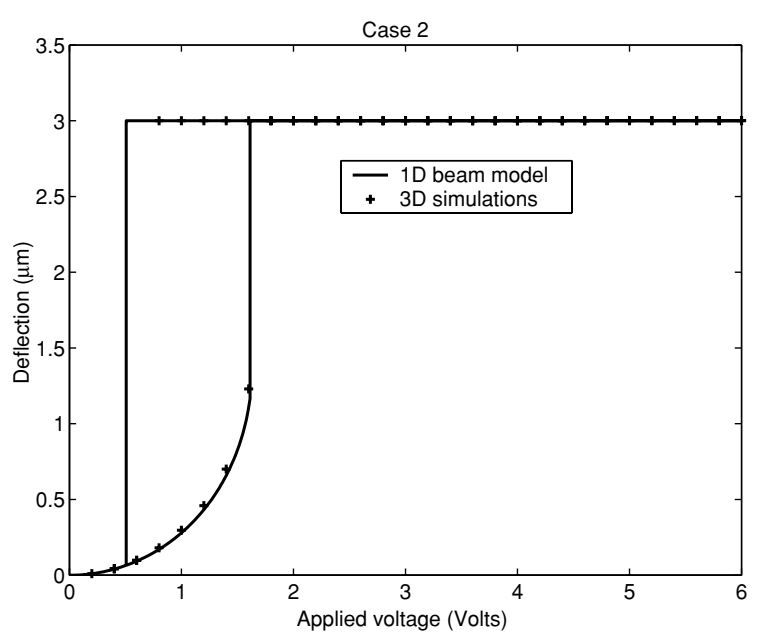

Figure 13. Variation of the moving electrode tip deflection with the applied voltage for the Case 2 example.

is verified in CoventorWare ${ }^{\mathrm{TM}}$ by finding out the solutions for various initial deflection conditions of the moving electrode. For instance, figure 11 shows all the three possible states for the Case 2 example at $1.5 \mathrm{~V}$.

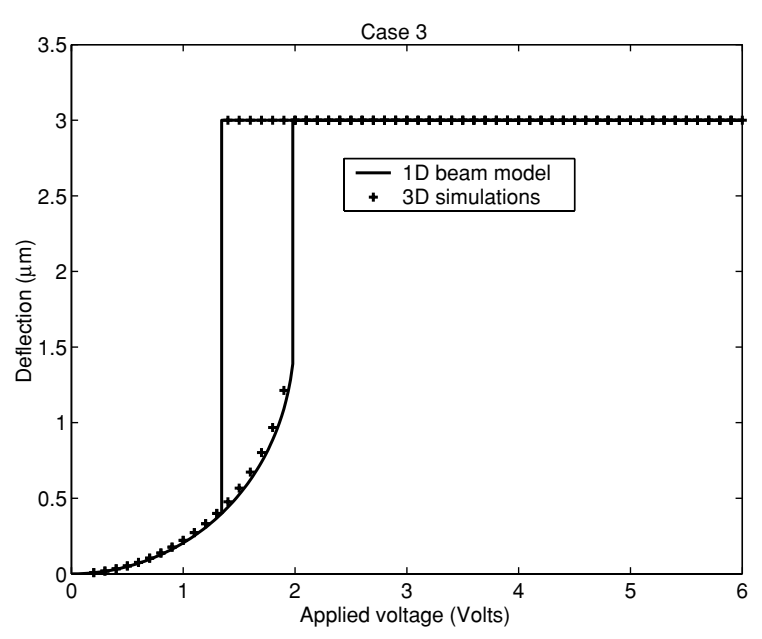

Figure 14. Variation of the moving electrode tip deflection with the applied voltage for the Case 3 example.

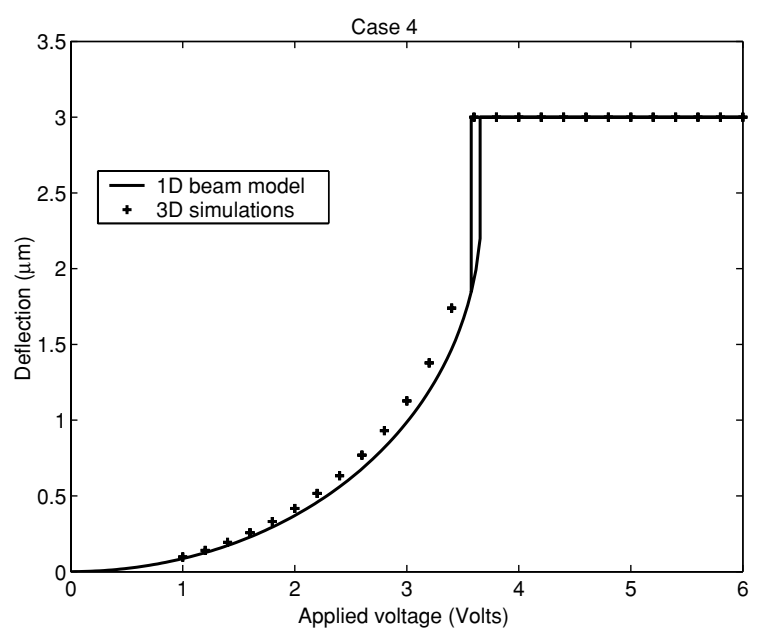

Figure 15. Variation of the moving electrode tip deflection with the applied voltage for the Case 4 example.

The variation of the moving electrode tip deflection with the applied voltage is shown in figures 12-16 for all the examples. Figure 17 shows the variation in the contact length with $V$ for the Case 1 example. Note that the scaling law gives a very good approximation for this example even for the $3 \mathrm{D}$ model. 


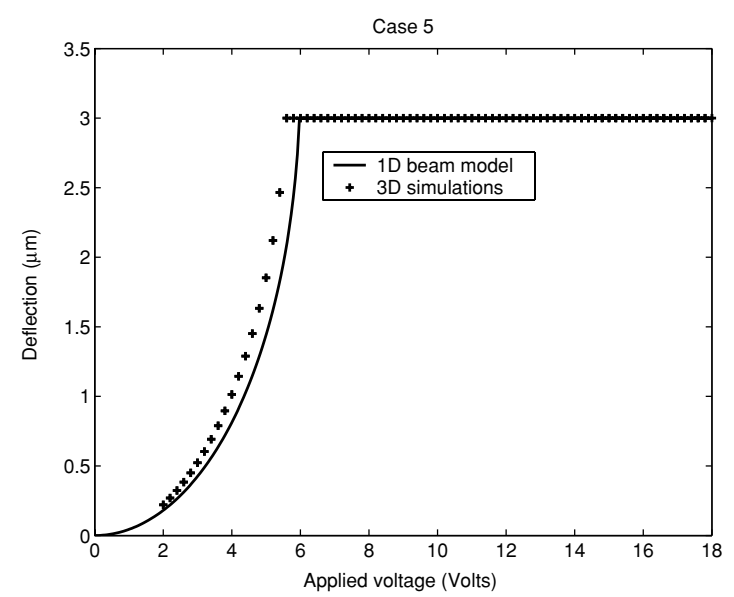

Figure 16. Variation of the moving electrode tip deflection with the applied voltage for the Case 5 example.

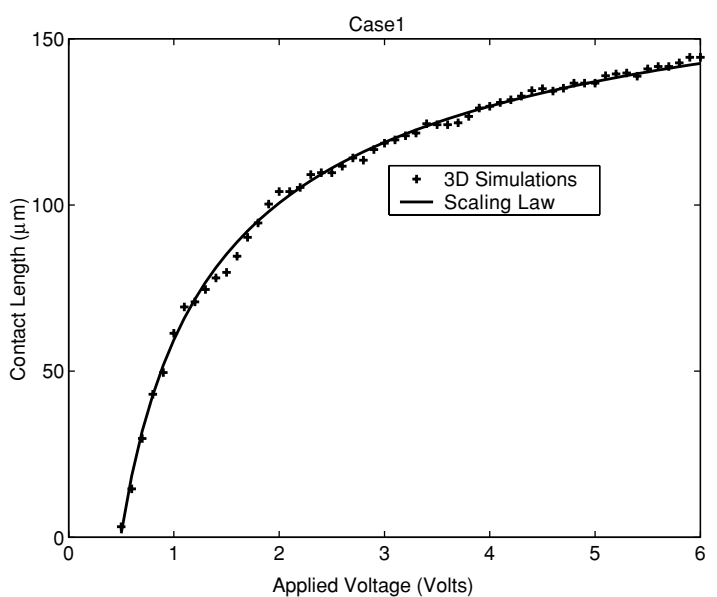

Figure 17. Variation of the moving electrode contact length with the applied voltage for the Case 1 example.

\section{Conclusions}

Cantilever beam electrostatic actuators with an intermediate dielectric layer have been analyzed in detail over the entire operational range using a beam model. The results are validated with the $3 \mathrm{D}$ simulation results from CoventorWare $^{\mathrm{TM}}$.

Three qualitatively different configurations, here called floating, pinned and flat, have been identified and studied. A scaling law is found for the flat configuration. Transitions from and to the floating configuration (pull-in and pull-out) and transitions from pinned to flat (pull-down) and flat to pinned (pull-up) have been studied as well. Bi-stable and tri-stable states have been found. A classification of all possible types of transitions is made based on the dielectric layer parameters. Dynamic stability analyses have complemented the study of these configurations and transitions. Higher dielectric thickness gives more regular and predictable behavior at the cost of lower overall tunability in device characteristics.

\section{Acknowledgments}

The authors thank the National MEMS Design Centre (NMDC), funded by the National Program on Smart Materials
(NPSM) for access to CoventorWare ${ }^{\mathrm{TM}}$. The authors also thank G K Ananthasuresh for valuable comments.

\section{References}

[1] Rocha L A, Cretu E and Wolffenbuttel R F 2004 Full characterisation of pull-in in single-sided clamped beams Sensors Actuators A 110 301-9

[2] Shrivastava V, Aluru N R and Mukherjee S 2004 Numerical analysis of 3D electrostatics of deformable conductors using a Lagrangian approach Eng. Anal. Bound. Elem. 28 583-91

[3] Younis M I, Abdel-Rahman E M and Nayfeh A H 2003 A reduced-order model for electrically actuated microbeambased MEMS IEEE J. Microelectromech. Syst. 12 672-80

[4] Rebeiz G M 2003 RF MEMS: Theory, Design and Technology (New Jersey: Wiley)

[5] Gupta R K 1997 Electrostatic pull-in test structure design for in-situ mechanical property measurements of microelectromechanical systems (MEMS) PhD Thesis Massachusetts Institute of Technology

[6] Hung E S and Senturia S D 1999 Generating efficient dynamic models for microelectromechanical systems from a few finite element simulation runs IEEE J. Microelectromech. Syst. 8 280-9

[7] Gabby L D, Mehner J E and Senturia S D 2000 Computer-aided generation of reduced-order dynamic macromodels-I: non-stress-stiffened case IEEE J. Microelectromech. Syst. 9 262-9

[8] Pelesko J A 2002 Mathematical modeling of electrostatic MEMS with tailored dielectric properties SIAM J. Appl. Math. 62 888-908

[9] Goldsmith C, Randall J, Eshelman S, Lin T H, Denniston D, Chen S and Norvell B 1996 Characteristics of micromachined switches at microwave frequencies IEEE MTT-S Int. Microwave Symp. Digest vol 2 (San Francisco, CA) pp 1141-4

[10] Muldavin J B and Rebeiz G M 2000 High-isolation CPW MEMS shunt switches. 1. Modeling IEEE Trans. Microwave Theory Tech. 48 1045-52

[11] Hung E S and Senturia S D 1998 Tunable capacitors with programmable capacitance-voltage characteristic Solid-State Sensors and Actuators Workshop pp 292-5

[12] Ionis G V, Dec A and Suyama K 2001 A zipper-action differential micro-mechanical tunable capacitor Proc. MEMS Conf. pp 24-6

[13] Ketterl T, Weller T and Fries D 2001 A micromachined tunable CPW resonator IEEE MTT-S Int. Microwave Symp. Digest vol 1 (Phoenix, AZ) pp 345-8

[14] Nathanson H C, Newell W E, Wickstrom R A and Davis J R 1967 The resonant gate transistor IEEE Trans. Electron Devices 14 117-33

[15] Tilmans H A C 1996 Equivalent circuit representation of electromechanical transducers: I. lumped-parameter systems J. Micromech. Microeng. 6 157-76

[16] Dec A and Suyama K 1998 Micromachined electro-mechanically tunable capacitors and their applications to RF IC's IEEE Trans. Microw. Theory Tech. 46 2587-96

[17] Pamidighantam S, Puers R, Baert K and Tilmans H A C 2002 Pull-in voltage analysis of electrostatically actuated beam structures with fixed-fixed and fixed-free end conditions J. Micromech. Microeng. 12 458-64

[18] Gilbert J R, Legtenberg R and Senturia S D 1995 3D coupled electromechanics for MEMS: applications of CoSolve-EM Proc. Int. Conf. on MEMS (Amsterdam, The Netherlands) pp 122-7

[19] Gilbert J R, Ananthasuresh G K and Senturia S D 1996 3D modeling of contact problems and hysteresis in coupled electro-mechanics Proc. 9th Int. Workshop on Microelectromechanical Systems pp 127-32 
[20] Mastrangelo C H and Hsu C H 1992 A simple experimental technique for the measurement of the work of adhesion of microstructures Tech. Dig.-IEEE Solid-State Sensor and Actuator Workshop pp 208-12

[21] de Boer M P and Michalske T A 1999 Accurate method for determining adhesion of cantilever beams J. Appl. Phys. $86817-27$

[22] Chan E K L 1999 Characterization and modeling of electrostatically actuated polysilicon micromechanical devices PhD Thesis Stanford University
[23] Press W H, Teukolsky S A, Vetterling W T and Flannery B P 1992 Numerical Recipes in C: The Art of Scientific Computing (Cambridge: Cambridge University Press)

[24] Chan E K, Garikipati K and Dutton R W 1999 Characterization of contact electromechanics through capacitance-voltage measurements and simulations IEEE J. Microelectromech. Syst. 8 208-17

[25] Hu Y C, Chang C M and Haung S C 2004 Some design considerations on the electrostatically actuated microstructures Sensors Actuators A 112 155-61 